\title{
Exploration of Multiple Roots for a Polynomial System
}

\author{
Crina Grosan and Ajith Abraham \\ Faculty of Information Technology, Mathematics and Electrical Engineering, \\ Centre for Quantifiable Quality of Service in Communication Systems, Centre of Excellence \\ Norwegian University of Science and Technology, Trondheim, Norway \\ cgrosan@cs.ubbcluj.ro,ajith.abraham@ieee.org
}

\begin{abstract}
Several problems from engineering, chemistry, medicine, etc. can be formulated as a system of equations. Finding a solution for such a system sometimes requires high computational efforts. There are situations when these systems have multiple solutions. For such problems, the task is to find as many solutions as possible. In this paper, we deal with such systems of equations, which have multiple solutions and we attempt to solve them using two different approaches. Both approaches transform the problem into an optimization problem. The two approaches proposed in are (1) a modified line search and (2) an evolutionary algorithm. Several experiments are performed in order to emphasize the advantages and disadvantages of the two methods.
\end{abstract}

\section{Introduction}

A nonlinear system of equations is defined as:

$$
f(x)=\left[\begin{array}{c}
f_{1}(x) \\
f_{2}(x) \\
\vdots \\
f_{n}(x)
\end{array}\right]
$$

where $x=\left(x_{1}, x_{2}, \ldots, x_{n}\right), f_{1}, \ldots, f_{n}$ are nonlinear functions in the space of all real valued continuous functions on $\Omega=\prod_{i=1}^{n}\left[a_{i}, b_{i}\right] \subset \Re^{n}$

Some of the equations can be linear, but not all of them. Finding a solution for a nonlinear system of equations $f(x)$ involves finding a solution such that every equation in the nonlinear system is 0 :

$$
\left\{\begin{array}{c}
f_{1}\left(x_{1}, x_{2}, \ldots, x_{n}\right)=0 \\
f_{2}\left(x_{1}, x_{2}, \ldots, x_{n}\right)=0 \\
\vdots \\
f_{n}\left(x_{1}, x_{2}, \ldots, x_{n}\right)=0
\end{array}\right.
$$

The assumption is that a zero, or root, of the system exists. The solutions we are looking for are those points (if any) that are common to the zero contours of $f_{i}, i=1, \ldots, n$.

There are several ways to solve nonlinear equation systems [8]. Probably the most famous techniques are Newton type techniques. Other techniques are: Trust
Region method, Broyden method, Secant method, Halley method.

In most cases, the solution of a system of equations is not unique. Several practical problems require finding the multiple solutions for a system. In this research, we focus on these types of equations systems. For this purpose, we test two approaches, which treat the problem in two different ways. The first approach is a line search based technique able to obtain one approximate solution in one run. This technique is applied several times in order to get multiple solutions. The second approach transforms the system into a multiobjective optimization problem [4]. A population based metaheuristic (evolutionary algorithm) is then applied. Pareto dominance concept is used and a set of feasible solutions (Pareto optimal) are obtained in a single run. Rest of the paper is organized as follows. In Section 2, we present the two optimization approaches followed by experiment results in Section 3. Some Conclusions are provided towards the end.

\section{Optimization Approaches Used}

A modified line search and evolutionary algorithms are proposed for solving equation systems. Each of them and the way in which they treat the problem is presented below.

\subsection{Line search}

It is known that line search techniques uses a starting point. There are also versions, which allow the usage of multiple points and the search starts separately from each of these points. In the proposed approach, multiple arbitrary starting points are used. Each point is randomly generated over the definition domain $\left[\min _{1}, \max _{1}\right] \times\left[\min _{2}, \max _{2}\right] \times \ldots \times$ $\left[\min _{\mathrm{n}}, \max _{\mathrm{n}}\right]$.

For direction, we use a random value between -0.5 and 0.5 . The step value is $2+\frac{3}{2^{k^{2}}+1}$, where $k$ denotes the current iteration number. After a given number of iterations, the search process is restarted. In order to restart the algorithm, the best result obtained in the previous set of iterations is taken into account and by following the steps given below: 
- Among all the considered points, the solution for which the objective function is obtaining the best value is selected. If there are several such solutions, one of them is randomly selected. This solution will be a multi-dimension point in the search space and denoted by $x$ for an easier reference.

- For each dimension $i$ of the point $x$, the first partial derivative with respect to this dimension is calculated. This means the gradient of the objective function is calculated, which is denoted by $g$. Taking this into account, the bounds of the definition domain for each dimension is recalculated as follows:

$$
\begin{aligned}
& \text { if } g_{i}=\frac{\partial f}{\partial x_{i}}>0 \text { then } \max _{i}=x_{i} \\
& \text { if } g_{i}=\frac{\partial f}{\partial x_{i}}<0 \text { then } \min _{i}=x_{i}
\end{aligned}
$$

- The search process is re-started by re-initializing a new set of arbitrary points but between the newly obtained boundaries (between the new $\max _{\mathrm{i}}$ or new $\left.\min _{\mathrm{i}}\right)$.

The line search is a very useful optimization tool. The equations system is transformed into an optimization problem as follows [5][6][7]:

$$
\text { minimize } \sum_{i=1}^{n} f_{i}^{2}(x)
$$

\subsection{Evolutionary Approach}

The evolutionary approach transforms the systems of equations into a multiobjective optimization problem as follows:

$\operatorname{Minimize}\left[\begin{array}{c}a b s\left(f_{1}(x)\right) \\ a b s\left(f_{2}(x)\right) \\ \vdots \\ a b s\left(f_{n}(x)\right)\end{array}\right]$

Real encoding of solutions, tournament selection, convex crossover and Gaussian mutation are used [1][3][9][10]. Pareto dominance concept [10] is used in order to compare two solutions. A detailed description of the approach is given in [4].

\section{Experiments and Results}

We considered 4 systems of equations having between one and nine solutions [2]. In Table 1 the details of these systems are provided.

\begin{tabular}{|l|l|l|}
\hline \multicolumn{1}{|c|}{ Problem } & \multicolumn{1}{|c|}{$\begin{array}{c}\text { Number of } \\
\text { variables }\end{array}$} & \multicolumn{1}{|c|}{ Range } \\
\hline Brown & 5 & {$[-2,2]^{5}$} \\
\hline Bullard & 2 & $\begin{array}{l}{\left[5.49 \cdot e^{-6}, 4.553\right] \times[} \\
0.0021961,18.21]\end{array}$ \\
\hline Ferrais & 2 & {$[0.25,1] \times[1.5,6.28]$} \\
\hline Himmelblau & 2 & {$[-5,5]^{2}$} \\
\hline
\end{tabular}

Table 1. Benchmark functions used in experiments.

Each algorithm was run 10 times. All the non-dominated solution sets obtained were unified at the end of each run. For the line search (LS) approach, we considered all the solutions (out of the 10 obtained), which are different. Also, the evolution of the best merit function obtained by LS in all the 10 runs is illustrated. Parameters used by LS and EA for all benchmarks are given in Table 2 .

\subsection{Example 1 (Brown)}

This benchmark is given by the following system of equations:

$$
\left\{\begin{array}{l}
2 \cdot x_{1}+x_{2}+x_{3}+x_{4}+x_{5}-6=0 \\
x_{1}+2 \cdot x_{2}+x_{3}+x_{4}+x_{5}-6=0 \\
x_{1}+x_{2}+2 \cdot x_{3}+x_{4}+x_{5}-6=0 \\
x_{1}+x_{2}+x_{3}+2 \cdot x_{4}+x_{5}-6=0 \\
x_{1} \cdot x_{2} \cdot x_{3} \cdot x_{4} \cdot x_{5}-1=0
\end{array}\right.
$$

LS obtained a single exact solution in all the independent runs. This solution is $(1,1,1,1,1)$ for which the functions values are all equal to 0 . The evolution of the merit function is depicted in Figure 1. It can be seen that the merit function converges to 0 after 4 iterations (we consider 5 iterations in one re-start). This means there is not even a need to use derivatives and to restart the line search. The EA approach is obtaining multiple solutions but no one is closed to the result obtained by LS. In Figure 2 the solutions obtained by EA in all 10 runs are plotted. It can be observed that only few of them are having the Euclidian norm less than 1 .

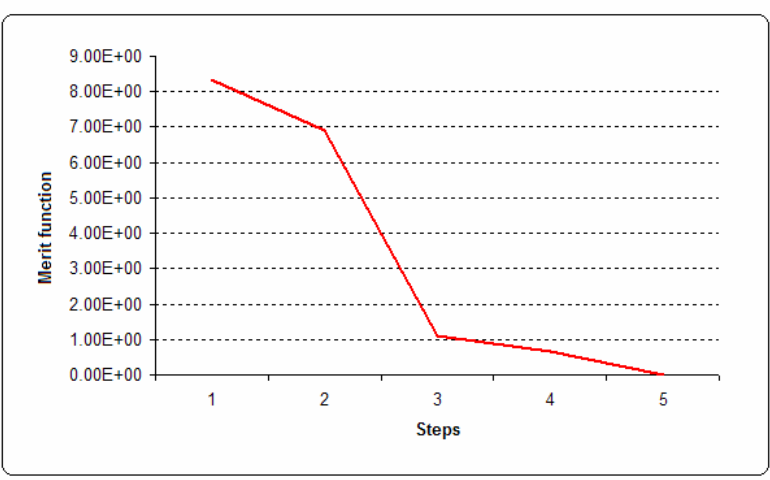

Figure 1. The evolution of the merit function for LS approach for the Brown benchmark. 


\begin{tabular}{|c|c|c|c|c|}
\hline Parameter settings & \multicolumn{4}{|c|}{ Parameter setting } \\
\hline & Brown & Bullard & Ferrais & Himmelblau \\
\hline \multicolumn{7}{|c|}{ Line search } & 100 & 100 \\
\hline No of starting points & 100 & 100 & 10 & 10 \\
\hline No of re-starts & 10 & 10 & 5 & 5 \\
\hline No of iterations per re-start & 5 & 5 & 100 & 500 \\
\hline \multicolumn{7}{|c|}{ Evolutionary algorithm } \\
\hline Population size & 500 & 100 & 500 & 500 \\
\hline Number of generations & 500 & 500 & 100 & 100 \\
\hline Size of nondominated set & 100 & 100 & 0.1 & 0.1 \\
\hline Sigma (for mutations) & 0.1 & 0.1 & 3 & 3 \\
\hline Tournament size & 3 & 3 & 3 & \\
\hline
\end{tabular}

Table 2. Parameters used in experiments by LS and EA

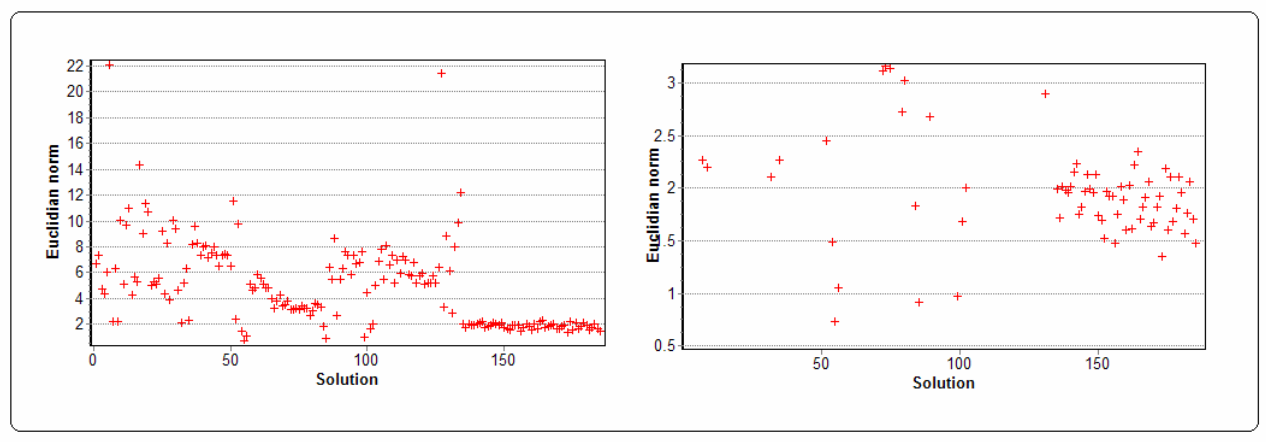

Figure 2. Solutions obtained by evolutionary algorithm for Brown benchmark.

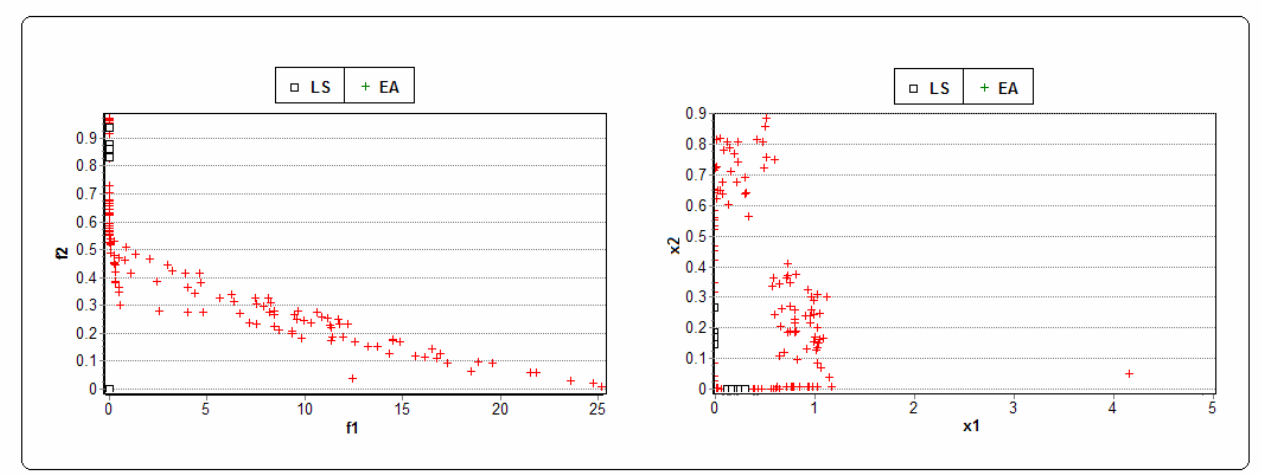

Figure 3. Solutions obtained by LS and EA for Bullard benchmark (objectives space-left, variables space-right).

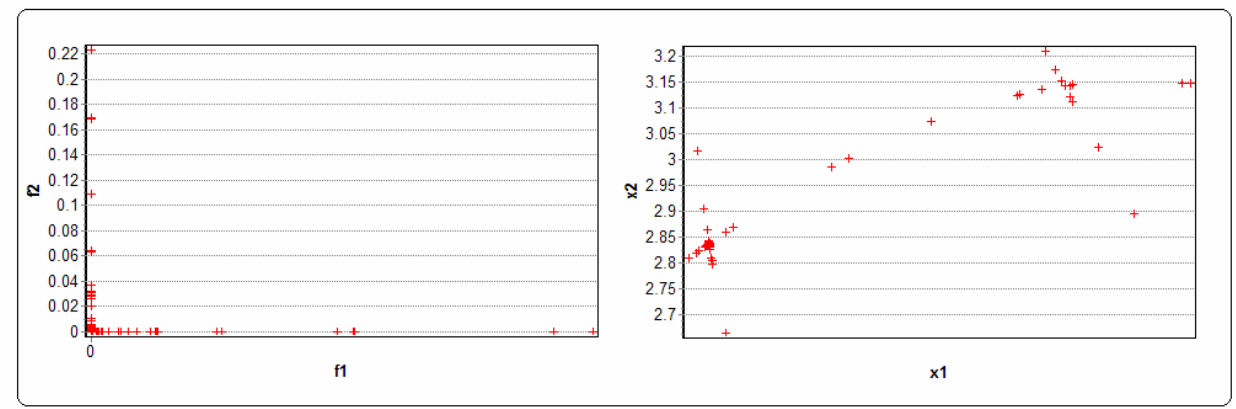

Figure 5. Solutions obtained by EA for Ferrais benchmark (objectives space - left, variables space-right). 


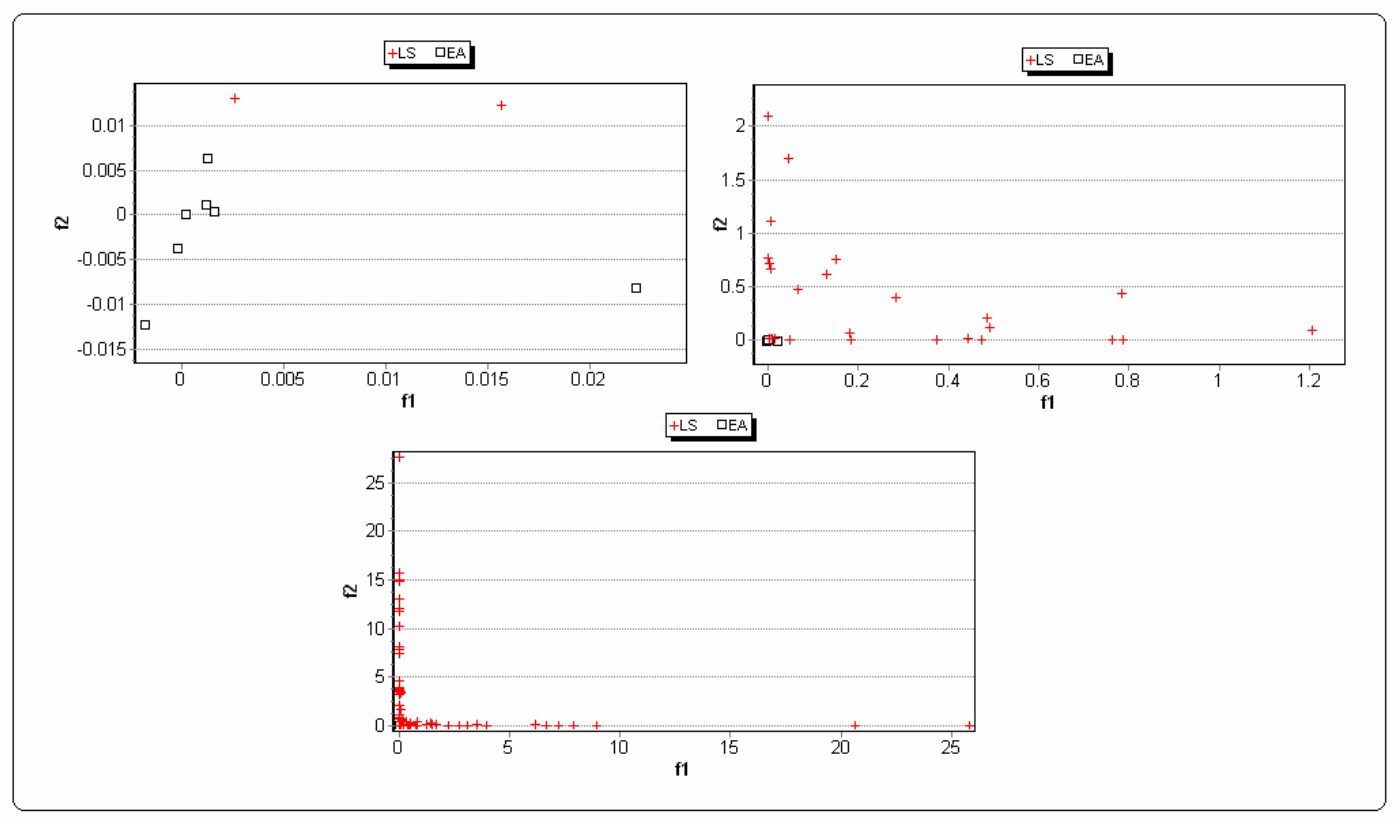

Figure 7. Solutions obtained by LS and EA for Himellblau benchmark represented in the objectives space (different sizes of the domain are considered for a better visualization).

\begin{tabular}{|l|l|l|l|}
\hline \multicolumn{2}{|c|}{ Solution } & \multicolumn{1}{c|}{ Functions values } \\
\hline \multicolumn{1}{|c|}{$\boldsymbol{x}_{\mathbf{1}}$} & \multicolumn{1}{c|}{$\boldsymbol{x}_{\mathbf{2}}$} & \multicolumn{1}{c|}{$\boldsymbol{f}_{\mathbf{1}}$} & \multicolumn{1}{c|}{$\boldsymbol{f}_{\mathbf{2}}$} \\
\hline-0.270841381989373 & -0.923036977925469 & $1.51325 \mathrm{E}-4$ & $4.19365 \mathrm{E}-5$ \\
\hline 0.0867085036783106 & 2.88423339327931 & $1.19474 \mathrm{E}-3$ & $1.212087 \mathrm{E}-3$ \\
\hline-3.07304526798170 & -0.0813371350117495 & $1.58640 \mathrm{E}-3$ & $3.61678 \mathrm{E}-4$ \\
\hline 3.38519412590167 & 0.0735892503227077 & $-1.93149 \mathrm{E}-4$ & $-3.80767 \mathrm{E}-3$ \\
\hline 3.00003916764214 & 1.99979209998351 & $1.25948 \mathrm{E}-3$ & $6.28423 \mathrm{E}-3$ \\
\hline 3.58441811907790 & -1.84770519568769 & $-1.85554 \mathrm{E}-3$ & $-1.22804 \mathrm{E}-2$ \\
\hline-0.127609787275348 & -1.95313642658286 & $2.22599 \mathrm{E}-2$ & $-8.22744 \mathrm{E}-3$ \\
\hline
\end{tabular}

Table 3. Solutions obtained by LS for Himmelblau benchmark.

\subsection{Example 2 (Bullard)}

This benchmark consists of a system of two equations given by:

$$
\left\{\begin{array}{l}
10000 \cdot x_{1} \cdot x_{2}-1=0 \\
e^{-x_{1}}+e^{-x_{2}}-1.001=0
\end{array}\right.
$$

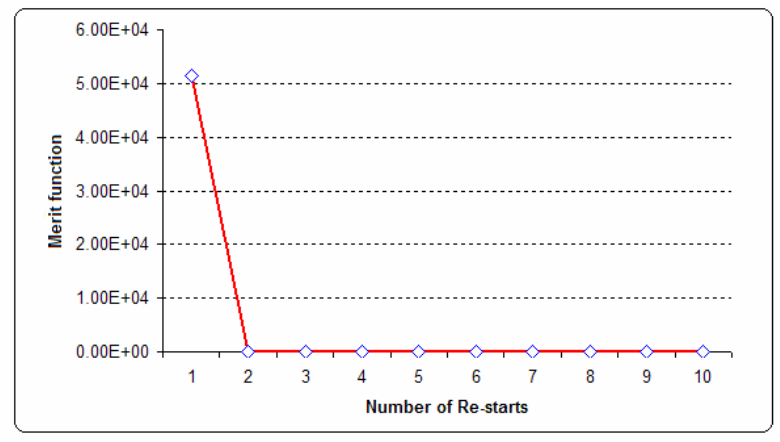

Figure 4. The evolution of merit function for LS approach for the Bullard benchmark.

\subsection{Example 3 (Ferrais)}

This benchmark consists of the following system of equations:

$$
\begin{aligned}
& \frac{0.25}{\pi} x_{2}+0.5 \cdot x_{1}-0.5 \cdot \sin \left(x_{1} \cdot x_{2}\right)=0 \\
& \frac{e}{\pi} \cdot x_{2}-2 \cdot e \cdot x_{1}+\left(1-\frac{0.25}{\pi}\right)\left(e^{2 \cdot x_{1}}-e\right)=0
\end{aligned}
$$

For this example, LS is obtaining a single solution which is $(0.5,3.14)$ for which the functions values are 0.0001265 and 0.0137805 .

Solutions obtained by EA are plotted in Figure 5. The evolution of the merit function for LS is depicted in Figure 6.

\subsection{Example 4 (Himmelblau)}

This example is given by the following system of equations:

$$
\left\{\begin{array}{l}
4 x_{1}^{3}+4 x_{1} x_{2}+2 x_{2}^{2}-42 x_{1}-14=0 \\
4 x_{2}^{3}+2 x_{1}^{2}+4 x_{1} x_{2}-26 x_{2}-22=0
\end{array}\right.
$$




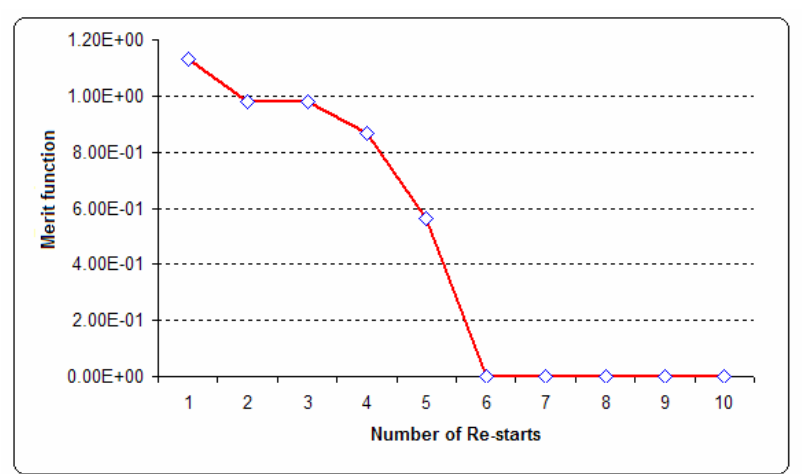

Figure 6. Evolution of merit function for LS for Ferrais benchmark.

There are 9 known solutions found so far. In 10 independent runs, LS is able to detect 7 solutions. The 7 solutions obtained by LS are given in Table 3. Solutions obtained by both LS and EA are depicted in Figure 7 (objectives space) and Figure 8 (variables space). As evident from Figure 8, in the variables space the solutions obtained by EA are centered on one of the solutions obtained by LS. The convergence of the merit function for the best result obtained in 10 runs (which is $0.2 \mathrm{E}-8$ ) is depicted in Figure 9.

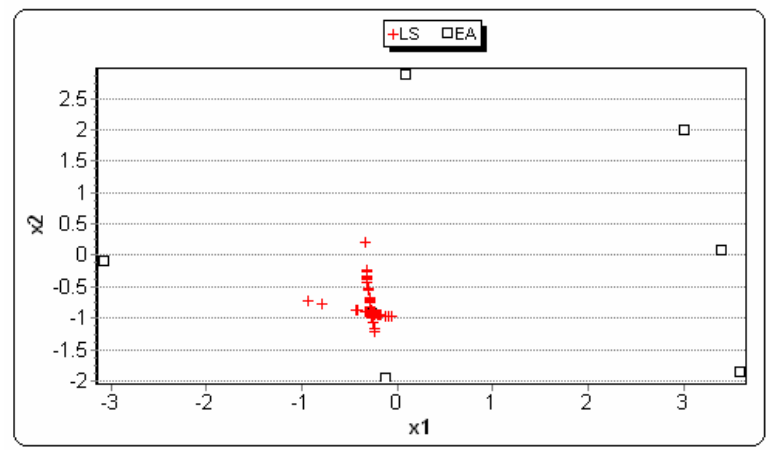

Figure 8. Solutions obtained by LS and EA for Himellblau benchmark represented in the variables space.

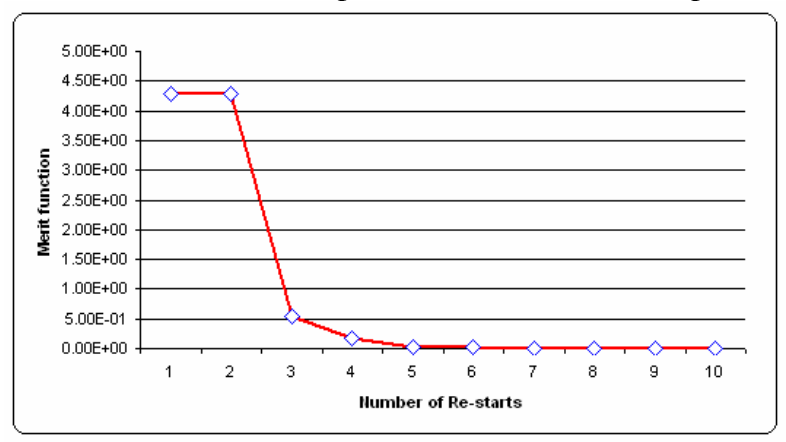

Figure 9. Evolution of merit function for LS for Himmelblau benchmark.

\section{Conclusions}

The goal of the paper is to find multiple solutions for a system of nonlinear equations. Two different techniques which transform the problem into an optimization problem are considered: line search based approach (LS), which transforms the systems of equations into a single objective optimization problem and Evolutionary Algorithms (EA) based approach which transforms the system into a multobjective optimization problem. Several equations systems having more than one solution are considered in experiments. The numerical results reveal that LS can approximate solutions better than EA even thought LS detect only one solution at one time and has to be applied multiple times while EA detects a set of solutions in one single run. Still the advantage of EA is that it can obtain multiple solutions and sometimes the user really needs a set from where can choose the desired solution.

\section{References}

[1] L.J. Eshelman, R.A. Caruna, J.D. Schaffer, Biases in the crossover landscape. In Proceeding of the Third International Conference on Genetic Algorithms, J. Schaffer (ed.), Morgan Kaufmann Publisher, Los Altos, CA, 10-19, 1989.

[2] C. A. Floudas, P.M. Pardalos, C. Adjiman, W.R. Esposito, Z. H. Gümüs, S. T. Harding, J. L. Klepeis, C.A. Meyer, C.A. Schweiger, Handbook of Test Problems in Local and Global Optimization (Nonconvex Optimization and Its Applications), Kluwer Academic Publishers, Dordrecht, 1999.

[3] D. E. Goldberg, Genetic algorithms in search, optimization and machine learning. Addison Wesley, Reading, MA, 1989.

[4] C. Grosan, A. and Abraham, A new approach for solving nonlinear equations systems, IEEE Transactions on Systems, Man and Cybernetics Part A, 2007 (in press).

[5] M.J. Hirsch , C.N. Meneses , P.M. Pardalos,M.G.C. Resende, Global optimization by continuous grasp, Optimization Letters, 1(2), 201-212, 2007.

[6] P.Y. Nie, A null space method for solving system of equations, Applied Mathematics and Computation, 149(1), 215-226, 2004.

[7] P.Y. NieAn SQP approach with line search for a system of nonlinear equations, Mathematical and Computer Modelling, 43, 368-373, 2006.

[8] J. M. Ortega and W. C. Rheinboldt, Iterative solution of nonlinear equations in several variables. New York: Academic Press, 1970

[9] W.M. Spears, K.A. De Jong, On the virtues of uniform crossover. In Proceedings of the Fourth International Conference on Genetic Algorithms, Morgan Kaufmann Publisher, 230-236, 1991.

[10]G. Syswerda, Uniform crossover in genetic algorithms. In Proceedings of the third Conference in Genetic Algorithms, J. Schaffer (ed.), Morgan Kaufmann Publisher, Los Altos, CA, 2-9, 1989.

[11]Steuer, R. E. Multiple Criteria Optimization. Theory, Computation, and Application. Wiley Series in Probability and Mathematical Statistics: Applied Probability and Statistics. New York: John Wiley Sons, Inc, 1986. 